\title{
Letter to the Editor concerning "Comparison of unilateral versus bilateral pedicle screw fixation in lumbar interbody fusion: a meta-analysis" by W. Ding et al. (2014) Eur Spine J 23(2):395-403
}

\author{
Guoping Liao ${ }^{1,2} \cdot$ Xing Feng $^{3} \cdot$ Tieshan Wang ${ }^{4} \cdot$ Lijing Chen $^{4} \cdot$ Jingming Lin $^{1}$
}

Received: 15 May 2015/Revised: 22 June 2015 / Accepted: 26 June 2015/Published online: 8 July 2015

(c) Springer-Verlag Berlin Heidelberg 2015

To the Editor,

In this meta-analysis, Ding et al. [1] compared the clinical results between unilateral and bilateral pedicle screw (PS) fixation in lumbar interbody fusion. They reached an important conclusion that unilateral PS fixation produced better results in operative time and blood loss in lumbar fusion, while no significant difference was found in hospital time, fusion rate and screw complications between the unilateral and bilateral PS fixation. It is a valuable study. Nevertheless, there are some comments we would like to raise related to this article.

To begin with, the investigators (Ding et al.) did not focus specifically or in any detail on the issue of the completeness of the search strategy report for databases, which plays an important role in meta-analysis. They just described the search strategy using the keywords such as, 'Spinal fusion', 'unilateral', 'bilateral' and 'pedicle screw'. We suggest that the investigators provide us a complete search strategy to strengthen the credibility of the metaanalysis.

Jingming Lin

liaoguoping1020@sina.com

1 Department of Pharmacy, Zhujiang Hospital of Southern Medical University, Guangzhou 510282, Guangdong, People's Republic of China

2 Changning Hospital of Traditional Chinese Medicine, Changning 421500, Hunan, People's Republic of China

3 Department of Pharmacy, The Medical College of Hunan Normal University, Changsha 410013, Hunan, People's Republic of China

4 Department of Pharmacy, Guangdong Women and Children Hospital and Health Institute, Guangzhou 511400, Guangdong Province, China
In addition, it was found that there were significant heterogeneities between studies in inoperative time, blood loss and hospital time in Figure $3\left(\mathrm{a}, I^{2}=95 \%\right.$; b, $\left.I^{2}=98 \% ; \mathrm{c}, I^{2}=99 \%\right)$. However, the investigators did not mention the heterogeneities. We suggest that the investigators should analyze the reasons for the significant heterogeneities. Meanwhile, the studies in inoperative time, blood loss and hospital time (Figure 3) were combined using the method of Inverse variance (IV) with the assumptions of a fixed-effects model, rather than DerSimonian and Laird random-effects model. In our opinion, the studies should be combined by DerSimonian and Laird random-effects model.

Eventually, we suggest that the publication bias should be assessed by some statistical tests (e.g., Egger's linear regression test or Begg's rank correlation test).

In a word, the investigators did not follow the Preferred Reporting Items for Systematic Reviews and Meta-Analyses (PRISMA). As a minor suggestion, adding PRISMA checklist and making some summary could make this manuscript better. We agree that unilateral PS fixation in lumbar fusion is as effective as bilateral PS fixation for lumbar degenerative diseases without major instability. In terms of operative time and blood loss, unilateral PS fixation even produces better results. This finding needs to be further confirmed by larger RCT with long-term follow-up.

Conflict of interest We have no competing interests to declare.

\section{Reference}

1. Ding WB, Chen YL, Liu H, Wang JR, Zheng ZM (2014) Comparison of unilateral versus bilateral pedicle screw fixation in lumbar interbody fusion: a meta-analysis. Eur Spine $\mathbf{J}$ 23(2):395-403 